\title{
Growth Kinetics of Lactic Acid Bacteria in Kunun-Zaki Supplemented with Soymilk
}

\author{
T. R. Duche* and S. Abdulganiu \\ Department of Microbiology, University of Agriculture, Makurdi-Nigeria \\ *Corresponding author
}

Keywords

Kunun-zaki,

Soymilk, Lactic

Acid Bacteria,

Supplementation,

Organoleptic

analysis,

Fermenting

Article Info

Accepted:

05 June 2020

Available Online:

10 July 2020

\section{A B S T R A C T}

Kunun-zaki are locally produced beverages which of recent have become popular among the various non-alcoholic drinks in the northern part of Nigeria; Viable bacterial counts were taken before and after the addition of soymilk on MRS agar plates after incubating anaerobically at $37{ }^{\circ} \mathrm{C}$ for 48 hours. Distinct colonies were purified for identification. kunun-zaki was fortified with soymilk, and subjected to sensory tests after $48 \mathrm{~h}$ of incubation. The effects of added soymilk and sensory quality attributes were evaluated. Furthermore, the sensory analysis showed a significant difference $(\mathrm{p}<0.05)$ observed between kunun- zaki and the one enriched with soymilk. Identification tests revealed the presence of Lactobacillus spp and Pediococcus spp as the major fermenting lactic acid bacteria. This result shows that adding soymilk to kunun-zaki that is about losing its aesthetic quality in a ratio of $50: 50$ can improve the overall taste, acceptability and nutritional benefits.

\section{Introduction}

Kunu is a milk-creamy non-alcoholic beverage sold in several public places including markets, offices, schools, motor parks and as drinks during festivities, weddings and naming ceremonies (Umaru et $a l ., 2014)$. It serves as an appetizer, food complement, and refresher to quench thirst (Oranusi et al., 2003; Amusa and Odunbaku, 2009; Amusa and Ashaye, 2009; Adelekan et al., 2013; Mbachu et al., 2014). Кипu is the generic name for all kinds of non-alcoholic beverages that are cereal-based with specifications usually attached to denote the base cereal grain (Nahemiah et al., 2014).

In Nigeria, particularly in the North Кипnи$z a k i$ is the most common lactic acid fermented nonalcoholic beverage that has wide acceptability among consumers due to its numerous advantages which include enhanced nutritional value, digestibility and many therapeutic benefits (Oranusi et al., 2003). 
The traditional methods of preparation of Kunnu-zaki have been described and there is general agreement on the dominance and beneficial effects of lactic acid bacteria (LAB) in the fermentation processes of this food (Odunfa, 2003; Teniola and Odunfa, 2003; Omemu et al., 2007). The LAB contributes to the enhancement of the organoleptic attributes of this food, as well as to its preservation and microbial safety (Hounhouigan et al., 2002; Caplice and Fitzgerald, 2001; Calderon et al., 2001). Lactic acid fermentation of cereal-based foods is a long age technology in African settings and has long been used in household processing of different foods (Mensah, 2002; Oyewole, 2002).

The primary antimicrobial effect exerted by LAB is the production of lactic acid and reduction of $\mathrm{pH}$ (Daeschel, 2003) which can antagonize the growth of some spoilage and pathogenic bacteria in foods. The antimicrobial producing LAB may be used as protective cultures to improve the microbial safety of foods and they also play an important role in the preservation of fermented foods, which is usually achieved by inhibition of contaminating spoilage bacteria such as Pseudomonas and pathogens such as Staphylococcus aureus, Salmonella spp. and Listeria monocytogenes (Buckenhuskes, 2001; Brinkten et al., 2002; Olasupo et al., 2003).

However, prolonged fermentation in the presence of LAB lowers the $\mathrm{pH}$ of the kunu, there by reducing the aesthetic and organoleptic quality. For fear of this, kunu is always consumed within a few hours of its production (maximum of $12 \mathrm{~h}$ ) after which it is considered spoiled in the absence of refrigeration (Adeleke and Abiodun, 2010). Food fortification is gaining prominence in recent years (Abulude et al., 2006) and Soymilk has been reported to be nutritious, containing about $44 \%$ protein, $28 \%$ carbohydrate $19 \%$ fat, $5.7 \%$ fiber and $4.4 \%$ ash, thus, enriching Kunun zaki with soymilk can be a good way of improving the macro and micronutrients content of the drink (Adepoju, 2012). It is in light of this that this work seeks to supplement kunun-zaki with soymilk after a long period of fermentation to improve the organoleptic quality and acceptability by consumers.

\section{Materials and Methods}

\section{Collection of samples and enumeration of lactic acid bacteria}

Traditionally prepared samples of fermented soymilk and kunnu-zaki were randomly obtained from ten (10) different food vendors at the University of Agriculture Makurdi, South Core. Samples were transported immediately to the Laboratory for enumeration and identification of lactic acid bacteria. After agitation for 2 minutes, samples were serially diluted. Aliquots $(0.1 \mathrm{ml})$ of each sample were plated out on De Man, Rogosa and Sharpe (MRS) agar and incubated anaerobically for 48 hours at $37^{\circ} \mathrm{C}$ for the enumeration of Lactic acid bacteria (LAB). Total viable counts ofthe LAB isolates were recorded as colony-forming units per $\mathrm{ml}(\mathrm{CFU} / \mathrm{ml})$ after 48hours of incubation. Changes in $\mathrm{pH}$ and titratable acidity (TTA) of samples were monitored every 12 hours for $48 \mathrm{~h}$ before and after the addition of soymilk.

\section{Identification of lactic acid bacteria}

After the enumeration of viable colonies, distinct ones were further picked based on morphology and sub-cultured repeatedly on MRS agar till pure colonies were obtained. Each 48-hour old bacteria culture on MRS Agar was Gram-stained and all Gram-positive isolates were taken for further identification. 


\section{Organoleptic analysis}

The sensory evaluation of the 10 supplemented kunun zaki with soymilk used in this study was done using a 7 point hedonic scale, where $1=$ like extremely, $2=$ like very much, 3=like slightly, $4=$ neither like nor dislike, 5=dislike, 6=dislike very much, $7=$ dislike extremely (Larmond, 1977) to measure the sensory qualities (appearance, taste, aroma, consistency and overall acceptability) of the product by using 10member panelist (comprising of lecturers, laboratory staff and students of the Department of Biological Sciences, Federal University of Agriculture, Makurdi). The panelists were instructed to rinse their mouth with clean water between the times of tastings

\section{Statistical analysis}

Data was analyzed using Analysis of Variance (ANOVA) to determine significant differences between the means and these were expressed as mean \pm standard deviation (SD). The level of significance was at $\mathrm{P}<0.05$. Statistical differences between means were compared using pair T-test.

\section{Results and Discussion}

Lactobacillus and Pediococcus species were isolated from 10 samples of Kunu-zaki. Inferences of these organisms were based on the morphological and physiological characteristics determined by the different biochemical tests as presented in table 1 .

Identification of these organisms from Kunuzaki was near in agreement with those isolated by Victor-Aduloju et al., (2018), although all their isolates based on probable identification were Lactobacillus spp. This result also conforms with the work of Okoronkwo (2014) who isolated similar organisms from Kunu-zaki and Burukutu.
Changes in $\mathrm{pH}$, titrable acidity and viable counts of LAB isolates during fermentation of non-supplemented kunu-zakiat varying time intervals from $0 \mathrm{~h}$ to $48 \mathrm{~h}$ are presented in table 2. From sample KZ1 to KZ10, there was a significant difference in the number of viable lactic acid bacterial (LAB) counts and the amount of titrable acidity (TTA) produced while the $\mathrm{pH}$ fluctuated between $3.30 \pm 0.10$ to $5.90 \pm 0.11$ without any statistical differences.

The only sample that showed a significant difference in the amount of TTA $(0.51 \pm 0.01)$ and LAB count $\left(6.0 \times 10^{5} \pm 5.00\right)$ in between the time intervals of 0 to $12 \mathrm{~h}$ fermentation was KZ1. This result is in contrast with that of Braide et al., (2018) where all their results showed high $\mathrm{pH}$ values which were directly proportional to the titrable acidity.

The differences in our results may be due to the duration of fermentation since all our samples were purchased from vendors without any standard production process. The increase at the initial time could be attributed to the utilization of nutrients in the medium. The decrease in initial high count of lactic acid in table 2 could be as a result of nutrient depletion in the slurry. Analysis of variance shows a significant difference between the $\mathrm{pH}$, lactic acid bacteria counts and titratable acidity (TTA) during the fermentation at $\mathrm{P}<$ 0.05 . The decrease in lactic acid bacteria could be as a result of the depletion of nutrients during fermentation. Although LAB are acidophilic, high concentrations of free acids can result in the production of growth inhibitors. Therefore, the high colony counts recorded were as a result of the low TTA observed. This result is following the one presented by Victor-Aduloju et al., (2018) where all the isolates grew well when the $\mathrm{pH}$ was at 5. As the $\mathrm{pH}$ decreased in the kunuzaki samples from $12 \mathrm{~h}$ up to $48 \mathrm{~h}$, bacterial colony count decreased significantly. 
Table.1 Morphological and Physiological Properties of Lactic Acid Bacteria (Lab) Isolated From the Kunun Zaki Supplemented with Soymilk

\begin{tabular}{|c|c|c|c|c|c|c|c|c|c|c|c|}
\hline S/N0. & Samples & $\begin{array}{c}\text { Characteristic } \\
\text { of Bacteria } \\
\text { Isolates }\end{array}$ & $\begin{array}{c}\text { Cell forms and } \\
\text { Their } \\
\text { Arrangement }\end{array}$ & $\begin{array}{c}\text { Gram } \\
\text { Reaction }\end{array}$ & $\begin{array}{c}\text { Motility } \\
\text { Test }\end{array}$ & $\begin{array}{c}\text { Catalase } \\
\text { Test }\end{array}$ & $\begin{array}{c}\text { Oxidase } \\
\text { Test }\end{array}$ & $\begin{array}{c}\text { Urease } \\
\text { Test }\end{array}$ & $\begin{array}{c}\text { Indole } \\
\text { Test }\end{array}$ & $\begin{array}{c}\text { Sugar } \\
\text { Fermentation } \\
\text { Test }\end{array}$ & $\begin{array}{l}\text { Suspected } \\
\text { Organism }\end{array}$ \\
\hline 1 & $\mathbf{A}$ & $\begin{array}{c}\text { Creamy, } \tan \& \\
\text { white }\end{array}$ & Cell in cluster & + & - & - & - & + & + & + & Lactobacillus \\
\hline 2 & B & $\checkmark$ & Rods & + & - & - & - & + & + & - & $\checkmark$ \\
\hline 3 & C & $\checkmark$ & $\begin{array}{l}\text { Short rod in } \\
\text { tetra or pairs }\end{array}$ & + & - & - & - & + & + & - & $\checkmark$ \\
\hline 4 & D & $\checkmark$ & $\begin{array}{l}\text { Cocci in pairs } \\
\text { and cluster }\end{array}$ & + & - & - & - & + & + & + & Pediococcus \\
\hline 5 & $\mathbf{E}$ & $\checkmark$ & $\begin{array}{l}\text { Short rod in } \\
\text { tetra or pairs }\end{array}$ & + & - & - & - & + & + & + & Lactobacillus \\
\hline 6 & $\mathbf{F}$ & $\checkmark$ & $\checkmark$ & + & - & - & - & + & + & + & $\checkmark$ \\
\hline 7 & G & $\checkmark$ & $\checkmark$ & + & - & - & - & - & + & - & $\checkmark$ \\
\hline 8 & H & $\checkmark$ & $\begin{array}{l}\text { Cocci in pairs } \\
\text { and cluster }\end{array}$ & + & - & - & - & + & - & + & Pediococcus \\
\hline 9 & I & $\checkmark$ & Long rod & + & - & - & - & + & + & - & Lactobacillus \\
\hline 10 & $\mathbf{J}$ & $\checkmark$ & $\begin{array}{l}\text { Long/short rod } \\
\text { in pairs and } \\
\text { singles }\end{array}$ & + & - & - & - & + & - & + & Lactobacillus \\
\hline
\end{tabular}


Table.2 Changes in $\mathrm{pH}$, TTA and Viable count of LAB during Fermentations of Kunun Zaki after every $12 \mathrm{~h}$

\begin{tabular}{|c|c|c|c|c|}
\hline Samples & Time & pH & TTA & LAB \\
\hline \multirow[t]{5}{*}{ KZ1 } & $\mathrm{Oh}$ & $5.86 \pm 0.01^{\mathrm{a}}$ & $0.03 \pm 0.01^{\mathrm{c}}$ & $9.4 \times 10^{5} \pm 5.00^{\mathrm{a}}$ \\
\hline & $12 \mathrm{~h}$ & $4.46 \pm 0.11^{\mathrm{a}}$ & $0.51 \pm 0.01^{\mathrm{a}}$ & $6.0 \times 10^{5} \pm 5.00^{b}$ \\
\hline & $24 \mathrm{~h}$ & $3.86 \pm 0.24^{\mathrm{a}}$ & $0.47 \pm 0.01^{\mathrm{c}}$ & $6.11 \times 10^{5} \pm 15.50^{a}$ \\
\hline & $36 \mathrm{~h}$ & $3.45 \pm 0.01^{\mathrm{a}}$ & $0.53 \pm 0.03^{\mathrm{c}}$ & $6.01 \times 10^{5} \pm 15.00^{\mathrm{a}}$ \\
\hline & $48 \mathrm{~h}$ & $3.30 \pm 0.10^{\mathrm{a}}$ & $0.59 \pm 0.01^{\mathrm{c}}$ & $5.01 \times 10^{5} \pm 5.00^{\mathrm{a}}$ \\
\hline \multirow[t]{5}{*}{ KZ2 } & $\mathrm{Oh}$ & $5.80 \pm 0.16^{\mathrm{a}}$ & $0.13 \pm 004^{b}$ & $9.0 \times 10^{5} \pm 5.00^{\mathrm{a}}$ \\
\hline & $12 \mathrm{~h}$ & $4.38 \pm 0.21^{\mathrm{a}}$ & $0.41 \pm 0.01^{\mathrm{b}}$ & $6.0 \times 10^{5} \pm 15.50^{\mathrm{a}}$ \\
\hline & $24 \mathrm{~h}$ & $3.81 \pm 0.25^{\mathrm{a}}$ & $0.37 \pm 004^{b}$ & $6.14 \times 10^{5} \pm 15.40^{a}$ \\
\hline & $36 \mathrm{~h}$ & $3.61 \pm 0.21^{\mathrm{a}}$ & $0.43 \pm 004^{b}$ & $6.01 \times 10^{5} \pm 5.00^{\mathrm{a}}$ \\
\hline & $48 \mathrm{~h}$ & $3.52 \pm 0.15^{\mathrm{a}}$ & $0.59 \pm 001^{b}$ & $5.01 \times 10^{5} \pm 5.00^{\mathrm{a}}$ \\
\hline \multirow[t]{5}{*}{ KZ3 } & $\mathrm{Oh}$ & $5.86 \pm 0.21^{\mathrm{a}}$ & $0.21 \pm 0.02^{\mathrm{a}}$ & $8.8 \times 10^{5} \pm 5.00^{c}$ \\
\hline & $12 \mathrm{~h}$ & $4.40 \pm 0.11^{\mathrm{a}}$ & $0.61 \pm 0.01^{\mathrm{a}}$ & $6.07 \times 10^{5} \pm 15.14^{c}$ \\
\hline & $24 \mathrm{~h}$ & $3.87 \pm 0.21^{\mathrm{a}}$ & $0.41 \pm 0.02^{\mathrm{a}}$ & $6.11 \times 10^{5} \pm 15.35^{c}$ \\
\hline & $36 \mathrm{~h}$ & $3.38 \pm 0.21^{\mathrm{a}}$ & $0.56 \pm 0.03^{\mathrm{a}}$ & $6.07 \times 10^{5} \pm 10.00^{c}$ \\
\hline & $48 \mathrm{~h}$ & $3.63 \pm 0.21^{\mathrm{a}}$ & $0.69 \pm 0.02^{\mathrm{a}}$ & $5.04 \times 10^{5} \pm 5.00^{c}$ \\
\hline \multirow[t]{5}{*}{ KZ4 } & $0 \mathrm{~h}$ & $5.85 \pm 0.17^{\mathrm{a}}$ & $0.03 \pm 0.01^{\mathrm{c}}$ & $6.0 \times 10^{5} \pm 5.00^{d}$ \\
\hline & $12 \mathrm{~h}$ & $4.45 \pm 0.21^{\mathrm{a}}$ & $0.51 \pm 0.01^{\mathrm{c}}$ & $5.56 \times 10^{5} \pm 15.32^{\mathrm{d}}$ \\
\hline & $24 \mathrm{~h}$ & $3.90 \pm 0.31^{\mathrm{a}}$ & $0.42 \pm 0.01^{\mathrm{c}}$ & $6.11 \times 10^{5} \pm 15.50^{d}$ \\
\hline & $36 h$ & $3.46 \pm 0.17^{\mathrm{a}}$ & $0.51 \pm 0.03^{\mathrm{c}}$ & $5.43 \times 10^{5} \pm 10.00^{d}$ \\
\hline & $48 \mathrm{~h}$ & $3.49 \pm 0.21^{\mathrm{a}}$ & $0.59 \pm 0.01^{\mathrm{c}}$ & $5.01 \times 10^{5} \pm 5.00^{d}$ \\
\hline \multirow[t]{5}{*}{ KZ5 } & Oh & $5.90 \pm 0.11^{\mathrm{a}}$ & $0.03 \pm 0.01^{\mathrm{c}}$ & $8.0 \times 10^{5} \pm 5.00^{c}$ \\
\hline & $12 \mathrm{~h}$ & $4.44 \pm 0.21^{\mathrm{a}}$ & $0.51 \pm 0.01^{\mathrm{c}}$ & $6.78 \times 10^{5} \pm 15.50^{c}$ \\
\hline & $24 \mathrm{~h}$ & $3.84 \pm 0.22^{\mathrm{a}}$ & $0.47 \pm 0.01^{\mathrm{c}}$ & $6.13 \times 10^{5} \pm 15.48^{c}$ \\
\hline & $36 \mathrm{~h}$ & $3.46 \pm 0.13^{\mathrm{a}}$ & $0.53 \pm 0.03^{\mathrm{c}}$ & $5.23 \times 10^{5} \pm 10.00^{c}$ \\
\hline & $48 \mathrm{~h}$ & $3.51 \pm 0.11^{\mathrm{a}}$ & $0.59 \pm 0.01^{\mathrm{c}}$ & $5.01 \times 10^{5} \pm 5.00^{c}$ \\
\hline \multirow[t]{5}{*}{ KZ6 } & $\mathrm{Oh}$ & $5.85 \pm 0.12^{\mathrm{a}}$ & $0.15 \pm 0.01^{b}$ & $9.0 \times 10^{5} \pm 5.00^{\mathrm{a}}$ \\
\hline & $12 \mathrm{~h}$ & $4.45 \pm 0.12^{\mathrm{a}}$ & $0.52 \pm 0.01^{\mathrm{b}}$ & $6.21 \times 10^{5} \pm 15.00^{a}$ \\
\hline & $24 \mathrm{~h}$ & $3.85 \pm 0.24^{\mathrm{a}}$ & $0.47 \pm 0.02^{b}$ & $6.11 \times 10^{5} \pm 15.43^{a}$ \\
\hline & $36 h$ & $3.89 \pm 0.12^{\mathrm{a}}$ & $0.50 \pm 0.01^{\mathrm{b}}$ & $5.01 \times 10^{5} \pm 10.00^{\mathrm{a}}$ \\
\hline & $48 \mathrm{~h}$ & $3.67 \pm 0.16^{\mathrm{a}}$ & $0.59 \pm 0.01^{\mathrm{b}}$ & $5.00 \times 10^{5} \pm 5.00^{\mathrm{a}}$ \\
\hline \multirow[t]{5}{*}{ KZ7 } & $0 \mathrm{~h}$ & $5.84 \pm 0.11^{\mathrm{a}}$ & $0.04 \pm 0.01^{\mathrm{c}}$ & $8.4 \times 10^{5} \pm 5.00^{c}$ \\
\hline & $12 \mathrm{~h}$ & $5.45 \pm 0.21^{\mathrm{a}}$ & $0.04 \pm 0.01^{\mathrm{c}}$ & $6.1 \times 10^{5} \pm 15.50^{c}$ \\
\hline & $24 \mathrm{~h}$ & $5.20 \pm 0.25^{\mathrm{a}}$ & $0.48 \pm 0.04^{\mathrm{c}}$ & $6.35 \times 10^{5} \pm 15.50^{c}$ \\
\hline & $36 \mathrm{~h}$ & $3.70 \pm 0.14^{\mathrm{a}}$ & $0.54 \pm 0.04^{c}$ & $5.04 \times 10^{5} \pm 10.00^{c}$ \\
\hline & $48 \mathrm{~h}$ & $4.30 \pm 0.23^{\mathrm{a}}$ & $0.60 \pm 0.01^{\mathrm{c}}$ & $5.50 \times 10^{5} \pm 5.00^{c}$ \\
\hline \multirow[t]{5}{*}{ KZ8 } & $0 \mathrm{~h}$ & $5.90 \pm 0.21^{\mathrm{a}}$ & $0.15 \pm 0.01^{b}$ & $6.8 \times 10^{5} \pm 5.00^{\mathrm{d}}$ \\
\hline & $12 \mathrm{~h}$ & $5.20 \pm 0.34^{\mathrm{a}}$ & $0.51 \pm 0.01^{\mathrm{b}}$ & $6.21 \times 10^{5} \pm 15.47^{d}$ \\
\hline & $24 \mathrm{~h}$ & $4.80 \pm 0.31^{\mathrm{a}}$ & $0.47 \pm 0.01^{\mathrm{b}}$ & $6.11 \times 10^{5} \pm 15.40^{d}$ \\
\hline & $36 \mathrm{~h}$ & $4.30 \pm 0.13^{\mathrm{a}}$ & $0.15 \pm 0.03^{b}$ & $5.01 \times 10^{5} \pm 10.00^{\mathrm{d}}$ \\
\hline & $48 \mathrm{~h}$ & $4.25 \pm 0.21^{\mathrm{a}}$ & $0.58 \pm 0.01^{\mathrm{b}}$ & $5.5 \times 10^{5} \pm 5.00^{d}$ \\
\hline \multirow[t]{5}{*}{ KZ9 } & $0 \mathrm{~h}$ & $5.85 \pm 0.11^{\mathrm{a}}$ & $0.03 \pm 0.00^{c}$ & $8.6 \times 10^{5} \pm 5.00^{c}$ \\
\hline & $12 \mathrm{~h}$ & $5.43 \pm 0.12^{\mathrm{a}}$ & $0.54 \pm 0.00^{c}$ & $6.32 \times 10^{5} \pm 15.33^{c}$ \\
\hline & $24 \mathrm{~h}$ & $4.66 \pm 0.26^{\mathrm{a}}$ & $0.47 \pm 0.01^{\mathrm{c}}$ & $6.11 \times 10^{5} \pm 15.45^{c}$ \\
\hline & $36 \mathrm{~h}$ & $4.03 \pm 0.11^{\mathrm{a}}$ & $0.52 \pm 0.03^{\mathrm{c}}$ & $5.01 \times 10^{5} \pm 10.00^{c}$ \\
\hline & $48 \mathrm{~h}$ & $3.64 \pm 0.13^{\mathrm{a}}$ & $0.59 \pm 0.01^{\mathrm{c}}$ & $5.01 \times 10^{5} \pm 5.00^{c}$ \\
\hline \multirow[t]{5}{*}{ KZ10 } & Oh & $5.83 \pm 0.01^{\mathrm{a}}$ & $0.15 \pm 0.01^{\mathrm{b}}$ & $8.0 \times 10^{5} \pm 5.00^{c}$ \\
\hline & $12 \mathrm{~h}$ & $5.46 \pm 0.03^{\mathrm{a}}$ & $0.52 \pm 0.01^{\mathrm{b}}$ & $6.20 \times 10^{5} \pm 15.50^{c}$ \\
\hline & $24 \mathrm{~h}$ & $5.12 \pm 0.21^{\mathrm{a}}$ & $0.49 \pm 0.03^{\mathrm{b}}$ & $6.19 \times 10^{5} \pm 5.00^{c}$ \\
\hline & $36 \mathrm{~h}$ & $4.76 \pm 0.14^{\mathrm{a}}$ & $0.51 \pm 0.03^{\mathrm{b}}$ & $5.03 \times 10^{5} \pm 5.00^{c}$ \\
\hline & $48 \mathrm{~h}$ & $3.87 \pm 0.13^{\mathrm{a}}$ & $0.59 \pm 0.01^{\mathrm{b}}$ & $5.01 \times 10^{5} \pm 5.00^{c}$ \\
\hline
\end{tabular}

Each data is the mean of triplicate determination with standard error. Means in the same column with different superscript are significantly different $(\mathrm{P}<0.05)$.

KEY: LAB. Lactic Acid Bacteria, $\mathrm{pH}=$ Hidrogen Potential and TTA $=$ Titratable Acidity 
Int.J.Curr.Microbiol.App.Sci (2020) 9(7): 583-592

Table.3 Changes in pH, TTA and Viable count of LAB during Fermentations of Kunun Zaki supplemented with soymilk after every $12 \mathrm{~h}$

\begin{tabular}{|c|c|c|c|c|}
\hline samples & Time & pH & TTA & LAB \\
\hline \multirow{5}{*}{ SKZ1 } & $0 \mathrm{~h}$ & $6.86 \pm 0.01^{\mathrm{a}}$ & $0.03 \pm 0.01^{\mathrm{c}}$ & $10.23 \times 10^{5} \pm 25.00^{a}$ \\
\hline & $12 \mathrm{~h}$ & $6.25 \pm 0.11^{\mathrm{a}}$ & $0.35 \pm 0.01^{\mathrm{a}}$ & $6.20 \times 10^{5} \pm 15.50^{\mathrm{a}}$ \\
\hline & $24 \mathrm{~h}$ & $5.86 \pm 0.24^{\mathrm{a}}$ & $0.37 \pm 0.01^{\mathrm{c}}$ & $7.15 \times 10^{5} \pm 16.00^{\mathrm{a}}$ \\
\hline & $36 \mathrm{~h}$ & $4.35 \pm 0.01^{\mathrm{a}}$ & $0.33 \pm 0.03^{\mathrm{c}}$ & $7.16 \times 10^{5} \pm 25.50^{\mathrm{a}}$ \\
\hline & $48 \mathrm{~h}$ & $4.30 \pm 0.10^{\mathrm{a}}$ & $0.42 \pm 0.01^{\mathrm{c}}$ & $9.01 \times 10^{5} \pm 25.00^{\mathrm{a}}$ \\
\hline \multirow[t]{5}{*}{ SKZ2 } & $\mathrm{Oh}$ & $6.40 \pm 0.16^{\mathrm{a}}$ & $0.03 \pm 004^{b}$ & $10.20 \times 10^{5} \pm 25.00^{\mathrm{a}}$ \\
\hline & $12 \mathrm{~h}$ & $6.12 \pm 0.21^{\mathrm{a}}$ & $0.13 \pm 0.01^{\mathrm{b}}$ & $8.15 \times 10^{5} \pm 6.50^{\mathrm{a}}$ \\
\hline & $24 \mathrm{~h}$ & $5.81 \pm 0.25^{\mathrm{a}}$ & $0.27 \pm 004^{b}$ & $7.10 \times 10^{5} \pm 16.01^{\mathrm{a}}$ \\
\hline & $36 \mathrm{~h}$ & $5.72 \pm 0.21^{\mathrm{a}}$ & $0.23 \pm 004^{b}$ & $6.30 \times 10^{5} \pm 25.50^{\mathrm{a}}$ \\
\hline & $48 \mathrm{~h}$ & $4.52 \pm 0.15^{\mathrm{a}}$ & $0.38 \pm 001^{\mathrm{b}}$ & $5.01 \times 10^{5} \pm 25.00^{\mathrm{a}}$ \\
\hline \multirow[t]{5}{*}{ SKZ3 } & $\mathrm{Oh}$ & $6.20 \pm 0.21^{\mathrm{a}}$ & $0.01 \pm 0.02^{\mathrm{a}}$ & $10.00 \times 10^{5} \pm 25.00^{\mathrm{a}}$ \\
\hline & $12 \mathrm{~h}$ & $5.80 \pm 0.11^{\mathrm{a}}$ & $0.21 \pm 0.01^{\mathrm{a}}$ & $8.15 \times 10^{5} \pm 6.50^{\mathrm{a}}$ \\
\hline & $24 \mathrm{~h}$ & $5.38 \pm 0.21^{\mathrm{a}}$ & $0.33 \pm 0.02^{\mathrm{a}}$ & $7.15 \times 10^{5} \pm 16.03^{\mathrm{a}}$ \\
\hline & $36 \mathrm{~h}$ & $4.38 \pm 0.21^{\mathrm{a}}$ & $0.36 \pm 0.03^{\mathrm{a}}$ & $6.16 \times 10^{5} \pm 25.00^{\mathrm{a}}$ \\
\hline & $48 \mathrm{~h}$ & $4.36 \pm 0.21^{\mathrm{a}}$ & $0.32 \pm 0.02^{\mathrm{a}}$ & $5.05 \times 10^{5} \pm 25.00^{\mathrm{a}}$ \\
\hline \multirow[t]{5}{*}{ SKZ4 } & $0 \mathrm{~h}$ & $6.25 \pm 0.17^{\mathrm{a}}$ & $0.03 \pm 0.01^{\mathrm{c}}$ & $8.00 \times 10^{5} \pm 25.00^{c}$ \\
\hline & $12 \mathrm{~h}$ & $4.45 \pm 0.21^{\mathrm{a}}$ & $0.09 \pm 0.01^{\mathrm{c}}$ & $7.40 \times 10^{5} \pm 25.00^{c}$ \\
\hline & $24 \mathrm{~h}$ & $4.10 \pm 0.31^{\mathrm{a}}$ & $0.12 \pm 0.01^{\mathrm{c}}$ & $7.15 \times 10^{5} \pm 16.02^{c}$ \\
\hline & $36 h$ & $3.96 \pm 0.17^{\mathrm{a}}$ & $0.21 \pm 0.03^{\mathrm{c}}$ & $6.16 \times 10^{5} \pm 25.00^{c}$ \\
\hline & $48 \mathrm{~h}$ & $3.57 \pm 0.21^{\mathrm{a}}$ & $0.32 \pm 0.01^{\mathrm{c}}$ & $5.03 \times 10^{5} \pm 25.00^{c}$ \\
\hline \multirow[t]{5}{*}{ SKZ5 } & $\mathrm{Oh}$ & $6.80 \pm 0.11^{\mathrm{a}}$ & $0.02 \pm 0.01^{\mathrm{c}}$ & $10.10 \times 10^{5} \pm 25.00^{\mathrm{a}}$ \\
\hline & $12 \mathrm{~h}$ & $6.74 \pm 0.21^{\mathrm{a}}$ & $0.31 \pm 0.01^{\mathrm{c}}$ & $8.15 \times 10^{5} \pm 6.50^{\mathrm{a}}$ \\
\hline & $24 \mathrm{~h}$ & $6.24 \pm 0.22^{\mathrm{a}}$ & $0.27 \pm 0.01^{\mathrm{c}}$ & $7.21 \times 10^{5} \pm 16.04^{\mathrm{a}}$ \\
\hline & $36 \mathrm{~h}$ & $5.16 \pm 0.13^{\mathrm{a}}$ & $0.33 \pm 0.03^{\mathrm{c}}$ & $6.16 \times 10^{5} \pm 25.00^{\mathrm{a}}$ \\
\hline & $48 \mathrm{~h}$ & $4.01 \pm 0.11^{\mathrm{a}}$ & $0.28 \pm 0.01^{\mathrm{c}}$ & $5.04 \times 10^{5} \pm 25.00^{\mathrm{a}}$ \\
\hline \multirow[t]{5}{*}{ SKZ6 } & $\mathrm{Oh}$ & $6.85 \pm 0.12^{\mathrm{a}}$ & $0.05 \pm 0.01^{\mathrm{b}}$ & $10.00 \times 10^{5} \pm 25.00^{\mathrm{a}}$ \\
\hline & $12 \mathrm{~h}$ & $6.45 \pm 0.12^{\mathrm{a}}$ & $0.17 \pm 0.01^{\mathrm{b}}$ & $8.15 \times 10^{5} \pm 6.50^{\mathrm{a}}$ \\
\hline & $24 \mathrm{~h}$ & $5.86 \pm 0.24^{\mathrm{a}}$ & $0.22 \pm 0.02^{b}$ & $7.35 \times 10^{5} \pm 16.00^{\mathrm{a}}$ \\
\hline & $36 \mathrm{~h}$ & $5.69 \pm 0.12^{\mathrm{a}}$ & $0.50 \pm 0.01^{\mathrm{b}}$ & $6.16 \times 10^{5} \pm 25.00^{\mathrm{a}}$ \\
\hline & $48 \mathrm{~h}$ & $4.67 \pm 0.16^{\mathrm{a}}$ & $0.51 \pm 0.01^{\mathrm{b}}$ & $5.11 \times 10^{5} \pm 25.00^{\mathrm{a}}$ \\
\hline \multirow[t]{5}{*}{ SKZ7 } & $0 \mathrm{~h}$ & $6.84 \pm 0.11^{\mathrm{a}}$ & $0.04 \pm 0.01^{\mathrm{c}}$ & $9.80 \times 10^{5} \pm 25.00^{b}$ \\
\hline & $12 \mathrm{~h}$ & $5.45 \pm 0.21^{\mathrm{a}}$ & $0.04 \pm 0.01^{\mathrm{c}}$ & $8.00 \times 10^{5} \pm 6.11^{\mathrm{b}}$ \\
\hline & $24 \mathrm{~h}$ & $5.20 \pm 0.25^{\mathrm{a}}$ & $0.38 \pm 0.04^{\mathrm{c}}$ & $7.21 \times 10^{5} \pm 16.03^{b}$ \\
\hline & $36 \mathrm{~h}$ & $4.90 \pm 0.14^{\mathrm{a}}$ & $0.44 \pm 0.04^{\mathrm{c}}$ & $6.16 \times 10^{5} \pm 25.00^{b}$ \\
\hline & $48 \mathrm{~h}$ & $4.30 \pm 0.23^{\mathrm{a}}$ & $0.50 \pm 0.01^{\mathrm{c}}$ & $5.01 \times 10^{5} \pm 25.00^{b}$ \\
\hline \multirow[t]{5}{*}{ SKZ8 } & $0 \mathrm{~h}$ & $6.90 \pm 0.21^{\mathrm{a}}$ & $0.15 \pm 0.01^{\mathrm{b}}$ & $10.10 \times 10^{5} \pm 25.00^{\mathrm{a}}$ \\
\hline & $12 \mathrm{~h}$ & $6.20 \pm 0.34^{\mathrm{a}}$ & $0.51 \pm 0.01^{\mathrm{b}}$ & $8.38 \times 10^{5} \pm 6.40^{\mathrm{a}}$ \\
\hline & $24 \mathrm{~h}$ & $5.80 \pm 0.31^{\mathrm{a}}$ & $0.47 \pm 0.01^{\mathrm{b}}$ & $7.09 \times 10^{5} \pm 16.01^{\mathrm{a}}$ \\
\hline & $36 \mathrm{~h}$ & $5.30 \pm 0.13^{\mathrm{a}}$ & $0.15 \pm 0.03^{b}$ & $6.16 \times 10^{5} \pm 25.00^{\mathrm{a}}$ \\
\hline & $48 \mathrm{~h}$ & $4.25 \pm 0.21^{\mathrm{a}}$ & $0.58 \pm 0.01^{\mathrm{b}}$ & $5.03 \times 10^{5} \pm 25.00^{\mathrm{a}}$ \\
\hline \multirow[t]{5}{*}{ SKZ9 } & $0 \mathrm{~h}$ & $5.85 \pm 0.11^{\mathrm{a}}$ & $0.03 \pm 0.00^{c}$ & $10.10 \times 10^{5} \pm 25.00^{\mathrm{a}}$ \\
\hline & $12 \mathrm{~h}$ & $5.63 \pm 0.12^{\mathrm{a}}$ & $0.04 \pm 0.00^{c}$ & $8.53 \times 10^{5} \pm 6.50^{\mathrm{a}}$ \\
\hline & $24 \mathrm{~h}$ & $5.60 \pm 0.26^{\mathrm{a}}$ & $0.07 \pm 0.01^{\mathrm{c}}$ & $7.15 \times 10^{5} \pm 16.00^{\mathrm{a}}$ \\
\hline & $36 \mathrm{~h}$ & $5.03 \pm 0.11^{\mathrm{a}}$ & $0.12 \pm 0.03^{\mathrm{c}}$ & $6.23 \times 10^{5} \pm 25.00^{\mathrm{a}}$ \\
\hline & $48 \mathrm{~h}$ & $4.64 \pm 0.13^{\mathrm{a}}$ & $0.39 \pm 0.01^{\mathrm{c}}$ & $5.05 \times 10^{5} \pm 25.00^{\mathrm{a}}$ \\
\hline \multirow[t]{5}{*}{ SKZ10 } & $0 \mathrm{~h}$ & $6.83 \pm 0.01^{\mathrm{a}}$ & $0.15 \pm 0.01^{\mathrm{b}}$ & $10.15 \times 10^{5} \pm 25.00^{\mathrm{a}}$ \\
\hline & $12 \mathrm{~h}$ & $6.46 \pm 0.03^{\mathrm{a}}$ & $0.12 \pm 0.01^{\mathrm{b}}$ & $8.39 \times 10^{5} \pm 6.43^{\mathrm{a}}$ \\
\hline & $24 \mathrm{~h}$ & $6.12 \pm 0.21^{\mathrm{a}}$ & $0.19 \pm 0.03^{b}$ & $7.15 \times 10^{5} \pm 16.02^{\mathrm{a}}$ \\
\hline & $36 \mathrm{~h}$ & $5.86 \pm 0.14^{\mathrm{a}}$ & $0.25 \pm 0.03^{b}$ & $6.11 \times 10^{5} \pm 25.00^{a}$ \\
\hline & $48 \mathrm{~h}$ & $4.47 \pm 0.13^{\mathrm{a}}$ & $0.39 \pm 0.01^{\mathrm{b}}$ & $5.00 \times 10^{5} \pm 25.00^{\mathrm{a}}$ \\
\hline
\end{tabular}

Each data is the mean of triplicate determination with standard error. Means in the same column with different superscript are significantly different $(\mathrm{P}<0.05)$ 


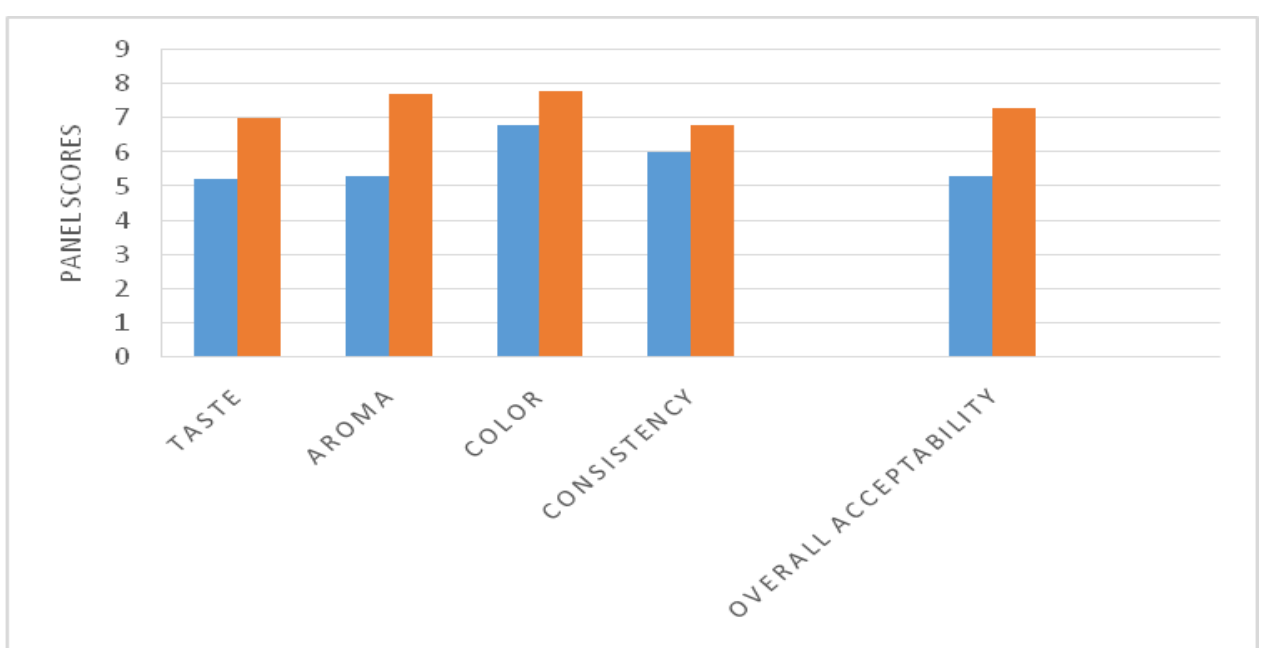

Fig.1 Organoleptic Scores of Kunun-zaki supplemented with and without Soymilk NKZ-non-supplemented kunun-zaki; SKZ- supplemented kunun-zaki

LAB are known to produce reasonable amounts of lactic acid when grown in a sugar enriched medium through the EmbdenMeyerhof fermentation pathway thereby lowering the $\mathrm{pH}$ and increasing the titrable acidity. There was a significant increase in the number of LAB counts askunun-zaki was fortified with soymilk (Table 3). It was observed that this fortification increased the $\mathrm{pH}$ values and subsequently reduced the TTA.

Analysis of variance showed significant differences between the treatment groups both in the bacterial counts and TTA but no significant difference occurred between the $\mathrm{pH}$ values of all treatments. The increase in growth at the initial time could be attributed to nutrients in the medium (Islamiyat et al., 2015). There was a progressive increase in $\mathrm{pH}$ and the sour taste decreased when soymilk was added to kunun-zaki that fermented for $48 \mathrm{~h}$ and its aroma became so appealing.

This condition may account for the proliferation of LAB (Table 3, Fig. 1).The result of the organoleptic test of kunun-zaki supplemented with soymilk significantly affected the overall acceptability (figure 1). Kunun-zaki supplemented with soymilk has the highest value in terms of aroma (7.7), colour (7.8) and overall acceptability of 7.3 compared to the non-supplemented kununzaki. The least preference for nonsupplemented kunu was 5.2 (taste). This result has some similarities with the results of Abulude et al., (2006) where different additives were added to kunu samples thereby making them organoleptically more acceptable.

Supplemented Kunun-zaki presents a more acceptable picture than the non-supplemented and is recommended as a functional food because of its nutritional value since soymilk is highly nutritious. This work showed that the desired attributes of kunu-zaki were enhanced when supplemented with soymilk thereby prolonging the shelf-life after $48 \mathrm{~h}$ of fermentation.

Soymilk can be used to improve the aesthetic nature of kunu that is getting out of taste but care should be taken when it comes to supplementation for safety, overall quality improvement and prolonged shelf life of kunu because most additives are harmful (Abulude et al., 2006). This study revealed the presence of lactic acid bacteria in the fermentation of cereals. Plain fermented kunun zaki and the one supplemented with 
soybean milk showed $L$. plantarum as the predominant organism and may be used as a starter culture in the production of kunu. This can also serve as a good source of probiotic and further studies should be conducted to evaluate this.

\section{Ethics approval}

The two authors of this manuscript have read and agreed to its content and are accountable for all aspects of the accuracy and integrity of the manuscript.

\section{Consent for publication}

This article is original and has not already being published in a Journal and is not currently under consideration by another Journal and none of the text, results or figure infringes upon the right of any third party. Therefore, the authors have conceded to the submission of this article for onward review and publication.

\section{Authors' contribution}

Abdulganiu Salihu was responsible for sample and data collection. He did all the experimental, analyzed and interpreted the data whileT. R. Duche was a major contributor in drafting the manuscript

\section{Availability of data and material}

The authors have authorized Willey the copyright to the article and all rights therein, including but not limited to the right to publish, republish, transmit, sell, distribute and otherwise use the article in whole or part in electronics and print editions of the journal and in derivative works throughout the world, in all languages and in all media of expression now known or later developed. The authors agree to the terms of Copyright and License Agreement and wish to retain the copyright of this article. Any third party is free to use this article free of charge provided its integrity is maintained.

\section{Abbreviations}

LAB - lactic acid bacteria

MRS - man rogosa sharpe agar

CFU - colony forming units

TTA - titrable acidity

ANOVA - analysis of variance

SD - standard deviation

$\mathrm{KZ}$ - kunun-zaki

SKZ - supplemented kunu-zaki

NKZ - non-supplemented kunu-zaki

\section{References}

Abulude Francis Olawale, Mary Omofolarin Ogunkoya and Vincent Adeniyi Oni, 2006. Mineral Composition, Shelflife and Sensory Attributes of Fortified Kunun-zaki beverage. Acta Sc. Pol., Technol. Aliment. 5 (1): 155-162

Adelekan, A.O., Alamu, A.E., Arisa, N.U., Adebayo, Y.O. and Dosa, A.S. 2013. Nutritional, Microbiological and Sensory Characteristics of Malted SoyKunuZaki: An Improved Traditional Beverage. Advances in Microbiology, 3:389-397.

Adeleke R.O. and Abiodun O.A. 2010. Physicochemical Properties of Commercial Local Beverages in Osun State, Nigeria. Pakistan Journal of Nutrition 9 (9): 853-855.

Adepoju, O T. 2012. Nutrient Composition, Anti-nutritional Factors and Contribution of Enriched Dry Guinea Corn (Sorghum sp) Leaf Extract to Nutrient Intake of Nigerian Consumers. International Conference

Environmental, Biomedical \& Biotechnology IPCBEE Aug 4-5, Dubai, 41: 23-27.
Amusa NA, Odunbaku O. A. 2009. 
Microbiological and Nutritional Quality of Hawked Kunu (A Sorghum Based Non-Alcoholic Beverage) widely consumed in Nigeria. Pakistan Journal Nutrition 8: 20-25.

Amusa, N.A. and Ashaye, O.A. 2009. Effect of Processing on Nutritional, Microbiological and Sensory Properties of Kunun-Zaki (A Sorghum Based NonAlcoholic Beverage) widely consumed in Nigeria. Pakistan Journal of Nutrition, 8: 288-292.

Braide W., Ukagwu N., Lugbe P. B. Akien Ali Al and Adeleye S. A. 2018. Chemical Properties and Microbiological Profile of Kunu zaki, A Non-Alcoholic Beverage. Biomed $J$. Sci. and Tech. Res. 4(1):1-5. DOI: 10.26717/BJSTR.2018.04.001001.

Brinkten, B., Minekns, M., Vossen, M. V., Leer, R. J. and Huisin't Veld, J. H. J. 2002. Antimicrobial activity of lactobacilli. Journal of Applied Bacteriology 77, 140-148.

Buckenhuskes, H. J. 2001. Selection criteria for lactic acid bacteria to be used as starter cultures for various food commodities. FEMS Microbiology Review 12, 253-272.

Calderon, M., Loiseau, G., and Guyot, J. P. 2001. Nutritional improvements and simplified cultivation medium to study growth and energetic of a sourdough lactic acid bacterium Lactobacillus fermentum Ogi during heterotactic fermentation of starch. Journal of Applied Microbiology 90, 1-9.

Caplice, E. and Fitzgerald, G. F. 2001. Food fermentations: Role of microorganisms in food production and preservation. International Journal of Food Microbiology 50, 131-149.

Daeschel, M. A. 2003. Antimicrobial substances from lactic acid bacteria for use as food preservatives. Food Technology, 43, 164-167.
Hounhouigan, D. J., Nout, M. J. R., Nago, C. M., Houben, J. H. and Rombouts, F. M. 2002. Characterization and frequency distribution of species of lactic acid bacteria involved in the processing of more, a fermented maize dough from Benin. International Journal of Food Microbiology 18, 115- 125.

Islamiyat Folashade Bolarinwa, Sulaiman Adebisi Olaniyan, Musibaudeen AbdulHammed and Maruff Olarewaju Oke 2015. Production and Quality Evaluation of Kunun-zaki (A Nigerian fermented cereal beverage) from Millet and Vigna-racemosa blends. Journal of Chemical and Pharmaceutical Research, 7(9): 347-352.

Mbachu A.E.,.Etok C.A., Agu K.C., Okafor O.I., Awah N.S., Chidi-Onuorah L.C., Ekwueme V.C., Okpala J., Ogbue M.O., and Ikele M.O. 2014. Microbial quality of kunu drink sold in Calabar, Cross River State, Nigeria. Journal of Global Bioscience, 3(2): 511-515.

Mensah, P. 2002. Fermentation - the key to food safety assurance in Africa. Food Control 8, 271-278.

Nehemiah, D., Bankole, O.S., Tswako, M.A., Nma- Usman K.I., Hassan H., and Fati, K.I. 2014. Hazard Analysis Critical Control Points in the Production of Soy-kununzaki: A Traditional Cereal-Based Fermented Beverage of Nigeria. American Journal of Food Science and Technology, 2(6): 196-202.

Odunfa, S. A. 2003. African fermented foods. In: Microbiology of Fermented Foods. Vol. 2. Wood B. J. (ed.). Elsevier Applied Science Publishers, London, and New York. pp. 35

Olasupo, N. A., Olukoya, D. K., and Odunfa, S. A. 2003. Studies on bacteriocinogenic Lactobacillus isolates from selected Nigerian fermented foods. Journal of Bacteriology and 
Microbiology 39, 181- 186.

Omemu, A. M., Oyewole, O. B. and Bankole, M. O. 2007. Significance of yeasts in the fermentation of maize for ogi production. Food Microbiology 24, 571576.

Ooronkwo, C. U. 2014. Isolation and Characterization of Lactic Acid Bacteria involved in the Fermentation of Millet and Sorghum sold in Nkwo-Achara Market, Abia State. Journal of Environmental Science, Toxicology and Food Technology, Volume 8(9):42-45.

Oranusi, S. U., Umoh, V. J., and Kwaga, J. K. P. 2003. Hazards and critical control points of Kunun-zaki, a non-alcoholic beverage in Northern Nigeria. Food Microbiology 20, 127-132.

Oyewole, O. B., 2002. Lactic fermented foods in Africa and their benefits. Food Control 8, 289-297.

Oyewole, O.A. and Isah P. 2012. Locally fermented foods in Nigeria and their significance to national economy: a review. Journal of Recent Advances in Agriculture, (4): 92 - 102.

Teniola, O. D. and Odunfa, S. A. 1995. Indigenous microflora of ogi and their role as spoilage microorganisms. The third Biennial Seminar of African fermented foods, Ghana. pp.71-76.

Umaru G.A., Tukur I.S., Akensire U.A, Adamu Z., Bello O.A., Shawulu A.H.B. and Audu, M. 2014. Microflora of Kunun-Zaki and Sobo drinks to public health in Jalingo Metropolis, NorthEastern Nigeria. International Journal of Food Research, 1: 1

Victor-Aduloju A. T., Anyamene C., Ogbu K. N., and Ishiwu C. N. 2018. Isolation and Identification of Probiotic Lactobacillus species from Traditional Drink Kununzaki fortified with Paddy Rice and Sweet Potatoes. African Journal of Food Sciences, Vol. 12 (9): 230-237.

\section{How to cite this article:}

Duche. T. R. and Abdulganiu. S. 2020. Growth Kinetics of Lactic Acid Bacteria in Kunun-Zaki Supplemented with Soymilk. Int.J.Curr.Microbiol.App.Sci. 9(07): 583-592. doi: https://doi.org/10.20546/ijcmas.2020.907.065 